\title{
Can nutraceuticals help in the control of plasma cholesterol levels?
}

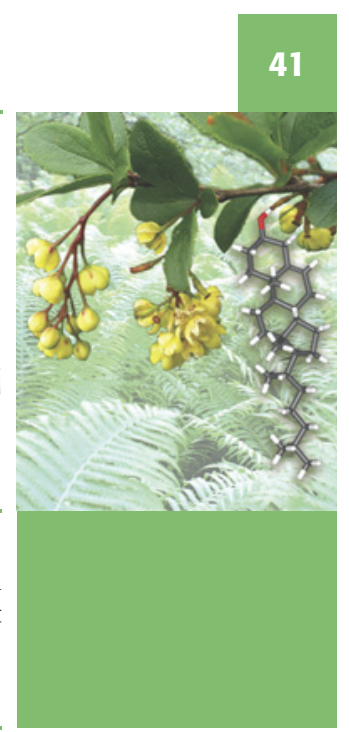

(C) Springer Healthcare - CEC Editore 2012

Should we propose the use of nutraceuticals in the control of plasma cholesterol and, hence, of cardiovascular risk? The answer is "Yes", especially if we accept the notion of "target" low-density lipoprotein cholesterol (LDL-c) in cardiovascular prevention. This concept leads to the identification of "ideal values" for LDL-c to be reached through therapeutic intervention; nutraceuticals may help achieve them.

In the most recent guidelines for LDL-c control in cardiovascular prevention, the target value decreases along with the increase in the so-called "global cardiovascular risk". In other words, lower values are to be reached when the overall probability of incurring cardiovascular events increases: the higher the risk, the lower the target value to be attained. As an example, the LDL-c target for a person at medium risk is $130 \mathrm{mg} / \mathrm{dl}$, but if the subject has had a myocardial infarction, his/her LDL-c needs to be lower than $100 \mathrm{mg} / \mathrm{dl}$ (ideally, 70-80 mg/dl). Conversely, in a low-risk person LDL-c concentrations up to $160 \mathrm{mg} / \mathrm{dl}$ are acceptable.

Such different goals translate into a diversified approach to LDL-c management. If the basal value of an individual only moderately exceeds his/her target, a dietetic intervention might correct the problem. If his/her distance from the target is higher than $10 \%$, diets need to be supplemented, e.g., with phytosterols, beta-glucan or soy protein (or combinations of them), to reach the desired goal. Stronger reductions call for pharmacological therapy. However, a combination of pharmacological and non-pharmacological treatments or the use of supplements containing natural statins, e.g., those with fermented red yeast, can easily reduce LDL-c by $20 \%$ as compared to basal values.

Based on the distribution-in an Italian populationof the individual distance from target and, thus, of the minimal reduction of LDL-c necessary to reach optimal values, it is possible to calculate the potential market of these products. In this respect, recent data from the CHECK study shed light on the profile of an Italian population (40-79 years) in terms of optimal target values and distance from them [1]. The CHECK data indicate that more than 30\% of the Italian population in that age range is not "at target". In absolute terms, we are talking about over 9 million people who should "do something" for their LDL-c. Approximately 50\% of these subjects, i.e., about 5 million, are $<20 \%$ from their target. Therefore, the use of appropriate diets and/or nutraceuticals might help them reach their goal without the need for drugs.

In order for these substances (see two relevant articles in this issue of NutraFoods) to be useful, some key requisites need to be fulfilled: 
- The active principle(s) should be chosen based on its (their) expected potency and the subject's distance from target.

- After some weeks of treatment, its efficacy (target reached or not?) must be verified. If the LDL-c target has not been reached, other interventions are to be planned.

- The use should be prolonged and regular (sporadic consumption is not effective in cardiovascular terms).

It goes without saying that such use of nutraceuticals and functional foods needs previous consultation and follow-up with a general practitioner or specialist.

Nutraceuticals can obviously be used also by "at target" persons: this will still reduce their (already low) risk and will contribute to the so-called "population strategy", which aims at shifting the entire population towards lower values of plasma cholesterol. This use also needs to be prolonged and regular, but it does not necessarily imply direct medical supervision. In summary, nutraceuticals aimed at cholesterol control might be helpful in bringing to target subjects who are not too far away from it, or fulfilling Geoffrey Rose's idea to reduce the whole population's cardiovascular risk. These are two important (and complementary) actions; therefore, let's use nutraceuticals mindfully and take advantage of their potentially interesting contribution to cardiovascular prevention.

\section{Andrea Poli Editor-in-Chief}

1. Poli A, Tragni E, Casula M et al. for the CHECK Group (2010) How many patients need statin treatment in a low-cardiovascular-risk country? Low-density lipoprotein-cholesterol target and distance from target distribution in an Italian cohort. Nutr Metab Cardiovasc Dis 22:327-336 